\title{
Secondary School Students' Knowledge and Opinions on Astrobiology Topics and Related Social Issues
}

\author{
Raquel Oreiro and Jordi Solbes
}

\begin{abstract}
Astrobiology is the study of the origin of life on Earth and the distribution of life in the Universe. Its multidisciplinary approach, social and philosophical implications, and appeal within the discipline and beyond make astrobiology a uniquely qualified subject for general science education. In this study, student knowledge and opinions on astrobiology topics were investigated. Eighty-nine students in their last year of compulsory education (age 15) completed a written questionnaire that consisted of 10 open questions on the topic of astrobiology. The results indicate that students have significant difficulties understanding the origin of life on Earth, despite exposure to the topic by way of the assigned textbooks. The students were often unaware of past or present achievements in the search for life within the Solar System and beyond, topics that are far less commonly seen in textbooks. Student questionnaire answers also indicated that students had problems in reasoning and critical thinking when asked for their opinions on issues such as the potential for life beyond Earth, the question of whether UFOs exist, or what our place is in the Universe. Astrobiology might help initiate student awareness as to current thinking on these matters and should be considered for general science education. Key Words: Astrobiology_-Students' views-Science education. Astrobiology 17, 91-99.
\end{abstract}

\section{Introduction}

A strobiology is a relatively recent discipline that has experienced exponential growth in recent years [see, e.g., Mix et al. (2006) and Des Marais et al. (2008) for a description of the field]. In the last 10-20 years, many important scientific advances have been achieved with regard to the origin of life on our planet and the search for life beyond Earth. This, in part, is due to advances in technology that have facilitated all aspects of space exploration.

Advances in the field of astrobiology have impacted not only the science community but the general public as well. Recent findings in astrobiology have become newsworthy and are often featured in popular films and books (Griffiths, 2004), which indicates that there is noticeable interest in astrobiology beyond the science community. Staley (2003) purported that this fascination has to do with "the great metaphysical questions of astrobiology" and the expectation that these questions may be answered in the near future; these authors also suggested that topics in astrobiology should be included in all levels of learning, including primary and secondary school.

A general interest in astrobiology is not the only point of importance when considering astrobiology from an educational perspective. As indicated by Rodrigues and Carrapiço
(2006), astrobiology's transdisciplinary approach allows for the integration of a number of areas of knowledge that traditionally have been kept apart and, hence, encourages a more holistic manner of science teaching; Tang (2005) also proposed the study of astrobiology as an integrative approach to be used in science education. Furthermore, astrobiology can serve as an excellent source of knowledge with which to discuss science overall, and the work-inprogress nature of astrobiology constitutes an example of how science, as a whole, operates. The potential utility of astrobiology in teaching the nature of science and employing critical thinking was also pointed out by Oliveira (2008).

Currently, there is a tendency toward cross-discipline training (Dinov, 2008). It is also important, however, to embrace the nature of science in the classroom and make use of science history and science and technology studies (STS) strategies to avoid a distorted view of science in its entirety, which could contribute to a general lack of interest in the sciences (Solbes and Vilches, 1997). Astrobiology would fit very well within the context of science and technology learning, as it could help students acquire a more realistic understanding of how science operates (Fergusson et al., 2012). Astrobiology has an origin that goes back to the ancient Greeks (Briot, 2013) and exemplifies STS relationships. 
Rodrigues and Carrapiço (2006) indicated that the larger obstacles for a proper inclusion of astrobiology in primary and secondary schools include the lack of educators trained in subjects that involve input from many disciplines, the growth of intelligent design, the difficulty of integrating astrobiology into the curriculum, and doubts and preconceptions of students about life and its limitations.

With regard to students' difficulties, as stated by Offerdah et al. (2003), there is a lack of research on the pre-instructional views and problems students may have when facing astrobiology topics, though it should be said that Offerdahl et al. (2003) focused mainly on environmental factors critical for life. Hansson and Redfors (2013) included similar questions in their study, among others in reference to exoplanets. Marques and Thompson (1997) considered the origin and nature of Earth and the development of life.

Oreiro and Solbes (2015) analyzed a set of textbooks to assess astrobiology content in Spanish secondary school curriculum. Although some related topics were found to be correctly identified, a considerable shortage of astrobiology content in textbooks was noted.

In the present study, a continuation of this work is presented, the objective of which was the analysis of student conceptions and views on various issues related to the origin of life on Earth and the search for life elsewhere.

\section{Methodology}

To discern student conceptions and views on topics in astrobiology, a questionnaire was designed and distributed to a total of 89 students from four different schools. Students were 15 years old on average and belonged to urban, Spanish schools.

\subsection{Objectives of teaching astrobiology}

The questionnaire covered varying components of astrobiology. In particular, the questionnaire was designed to investigate a set of teaching and learning objectives as defined in this study. These objectives serve as overarching guidelines for interpretive analysis, the results of which could help inform teaching program design.

Teaching and learning objectives that were defined include the following:

- O1. Understanding how life emerged and evolved on Earth.

- O2. Awareness of current achievements in the search for life within the Solar System.

- O3. Knowledge of the results and efforts in the search for extrasolar planets and intelligent forms of life.

- O4. Development of critical thinking concerning ufology and aliens.

- O5. Identification of the STS relationships of the field.

These objectives were devised by taking into account (i) the list of research topics in astrobiology as published by Burchell and Dartnell (2009), (ii) the example topic areas detailed by Staley (2003), (iii) the curricular proposal of Rodrigues and Carrapiço (2005), and (iv) key questions that guide the NASA hands-on activities within Astrobiology in Your Classroom: Life on Earth . and Elsewhere? (NASA, 2006). Most of these inputs can be easily incorporated as subcategories under one of the objectives defined.

\subsection{Instrument}

A written questionnaire was chosen as a tool to investigate student views on astrobiology and consisted of a set of 10 open questions that were intended to allow students to answer as freely as possible according to their personal views. With this aim, no multiple-choice options were provided. The preliminary questions were revised by other researchers from Valencia University (Spain) who are astronomy education or astrobiology experts. The authors sought approval from the Internal Review Board (IRB) of the University of Valencia. The IRB determined approval was not required, and a waiver was issued.

The questionnaire was first applied to a pilot group of 17 students. Based on the analysis of answers from the pilot phase, a Cronbach's alpha of 0.7 was obtained, which verifies the internal consistency of the questionnaire. Minor rephrasing and refinement of the design presented to the students was performed after the pilot phase analysis. The final questionnaire is shown in Table 1. Question 1 is similar to one used by Offerdahl et al. (2003), which reads " What elements are the most important for the existence of life?,' and question 2 is similar to one used by Marques and Thompson (1997), which has to do with inquiries about the relationship between the origin and nature of Earth and the origin and development of life.

Questions 1-3 in the questionnaire relate to life on Earth (O1), question 5 refers to the possibility of life within the Solar System (O2), and question 9 connects with objective 3 (O3). Critical thinking (O4) is investigated through questions 4, 6, and 8, and STS relationships (O5) are considered with questions 7 and 10 in Table 1.

\subsection{Student sample}

Compulsory school in Spain is organized into two periods: primary school (six levels, 6-12 years of age) and secondary school (four levels, 12-15 years of age). In the first stage, science is compulsory and structured within the broad subject Knowledge of the Social and Natural Environment. In this stage, basic astronomy and life-related issues are studied.

Table 1. Questionnaire for the Investigation of Student Views on Astrobiology

1. Name factors that make possible the existence of life on Earth.

2. If Earth was formed about $4500 \mathrm{My}$ ago, do you know when life started on our planet? (approx.)

2b. Do you know how it appeared?

3. What were the first living forms that emerged on Earth?

4. Draw an alien.

5. Name bodies of the solar system where life could exist or could have existed, besides Earth. Explain why.

6. Do you think there exist living forms in other places of the universe, outside our solar system? Why?

7. Do you think it is important to figure out whether intelligent civilizations exist anywhere? Why?

8. Do you believe UFOs exist? That is, intelligent beings visiting our planet? Justify the answer.

9. Do you know any real scientific project with the objective of establishing communication with possible extraterrestrial civilizations?

10. Why do you think we are in the universe? 
In the secondary school stage, the Natural Sciences subject is compulsory for three levels. A large amount of astronomy content is found in the first level (age 12) as follows: the Milky Way; stars and galaxies; properties of Solar System bodies; heliocentrism; and other relevant knowledge such as the importance of water, factors that make life possible on Earth, the history of life, and taxonomy. No other astrobiology-related content is found until fourth level.

In the fourth level of secondary school, the sciences are no longer compulsory, and students must choose among different propaedeutic itineraries. Within the science itinerary, Biology and Geology and Physics and Chemistry can be found as optional subjects. The former deals with Earth history, the origin of life on Earth, and first living beings and evolution.

We focused our study on students at the fourth level of secondary school following the science itinerary. This is their last year of compulsory school, and science subjects are optional. Students received important science background during their previous stages, and it is at this level that students specifically discuss the emergence of life on Earth.

The questionnaire in Table 1 was distributed to four groups of students that came from four different schools that were visiting the Sky Classroom, an educational project for the teaching of astronomy of the Astronomical Observatory in the University of Valencia (Ortiz Gil et al., 2005). The questionnaire was distributed prior to their visit. In total, 89 students from four public schools located in the Valencia (Spain) urban area participated in the experiment.

\subsection{Analysis}

An initial examination of all student answers was performed for each of the questions. This allowed us to organize the answers to each question in sets according to similarities, depth, or thematic patterns. In addition, categories were established that included one or more sets of answers to facilitate a global analysis of the students' performance.

Answers to most of the questions were categorized as follows: correct (C), partially correct (PC), or incorrect (I). The categories established for each question are detailed in Section 3. A few questions were analyzed differently, for example, when answers refl ed a personal opinion or view and it was diffi to defi what, per se, constituted correct from a common science point of view. These include questions 4,7 , and 10 , whose answers were nevertheless thematically grouped.

\section{Results}

Here, we present the results obtained for each of the questions in Table 1. A table containing the categories established and percentages of answers is provided for every item, together with a qualitative description.

\subsection{Factors that make possible the existence of life on Earth}

Table 2 summarizes the percentage of students with correct, partially correct, and incorrect answers. All students except one were able to name at least one factor that makes life on Earth possible. However, only $34.8 \%$ named three correct factors (C). For the rest, $47.2 \%$ of the students listed two factors (PC), and 18\% named one or no correct factor (I).
Table 2. Categories Defining Correct, Partially Correct, and Incorrect Answers to Item 1 of the Questionnaire

\begin{tabular}{lll}
\hline C & Name at least three correct factors & $34.8 \%$ \\
PC & Name two correct factors & $47.2 \%$ \\
I & Name one or no correct factor & $18.0 \%$ \\
\hline
\end{tabular}

The right column indicates the percentage of student answers within each category.

A large majority of students $(88.8 \%)$ identifi water as a fundamental element for life. The second most cited factor was the atmosphere, which was cited by $56.2 \%$ of the students, although the same concept is referred to by an additional $12.4 \%$ that answered with "the air." A suitable temperature was listed by $22.5 \%$ of the students, and only $19.1 \%$ indicated the correct distance between our planet and the Sun.

Among incorrect factors, oxygen stands out in that 53.9\% of the students listed it as an important factor for life, with answers such as "oxygen is very important for us," which speaks to an anthropocentric concept of life. Sunlight, or simply "the Sun," is another factor that appeared in $31.4 \%$ of the answers. Students also named plants (9\%); living beings (3.4\%); particular elements or molecules such as hydrogen $(6.7 \%)$, carbon $(3.4 \%)$, nitrogen $(3.4 \%)$, or carbon dioxide (2.2\%); and in less proportion soil, reproduction, salinity, altitude, and minerals, all considered incorrect within the context of this research.

"The factors that make possible life on Earth," as well as "The importance of water," are specific contents included in the official curriculum of the subject Natural Sciences in the first level (age 12).

\subsection{When and how life appeared on Earth}

Few students were able to respond properly to questions as to when life began on our planet (Table 3). Even when given Earth's age as a time reference, only $23.6 \%$ of the students indicated that life started between 4000 and 3000 My ago (C). Next, $14.6 \%$ specified that life started between 2500 and $1000 \mathrm{My}$, which is considered partially correct (PC) as at least the order of magnitude of time is correct. Another $61.8 \%$ of the students either did not answer or indicated that life started closer in time to the present. In general, it was observed that students did not have this issue clear, as we found many "Don't know" answers and tentative guesses such as "I imagine 100,000 years ago" or "Relatively short time ago, compared to 4500 My ago."

Students had difficulty explaining how life emerged on Earth (Table 4). Only $15.7 \%$ made reference to one of the plausible hypotheses about the origin of life on Earth. Mainly, students referred to the primordial soup hypothesis.

Table 3. Categories Defining Correct, Partially Correct, and Incorrect Answers to Item 2 of the Questionnaire

\begin{tabular}{lll}
\hline C & Date between 4000 and 3000 My ago & $23.6 \%$ \\
PC & Date between 2500 and 1000 My ago & $14.6 \%$ \\
I & Date closer than 1000 My ago & $61.8 \%$ \\
\hline
\end{tabular}

The right column indicates the percentage of student answers within each category. 
Table 4. Categories Defining Correct, Partially Correct, and Incorrect Answers to Item 2b of the Questionnaire

\begin{tabular}{lll}
\hline C & Explain one of the plausible options & $15.7 \%$ \\
PC & Only says it emerged in the water & $15.7 \%$ \\
I & Other, no answer, don't know & $68.5 \%$ \\
\hline
\end{tabular}

The right column indicates the percentage of student answers within each category.

Only three students mentioned the panspermia possibility. Another $15.7 \%$ of the answers were considered partially correct (PC), some of which asserted that life emerged in water from simple elements. The rest $(68.5 \%)$ either did not respond or were entirely incorrect. Incorrect answers were as follows: $7.9 \%$ of the students thought that life on Earth appeared due to, or after, the Big Bang, one student mentioned a supernova as the originator of life, and four students indicated that Adam and Eve were the starting point of life.

The Spanish official curriculum includes contents as "History of life" in the first year of Natural Sciences (age 12) and "Earth history" and "The origin of life" in the optional subject Biology and Geology (fourth level, age 15).

\subsection{The first living forms on Earth}

A total of $23.6 \%$ of the students gave a correct answer to this question (Table 5). Many students, $46 \%$, described the first living beings either as unicellular or microscopic (PC).

No answers, along with incorrect answers, made up the other $30.3 \%$. Most incorrect answers consisted of a description of simple beings, primitive or not much evolved living forms $(6.7 \%)$. However, other responses included the following: $4.5 \%$ thought that apes were the first living creatures, $4.5 \%$ responded that they were dinosaurs, $3.4 \%$ indicated plants, and one student thought that Adam and Eve were the first forms of life on Earth.

Students may, or may not, be taught about the first living beings on Earth in the first year of secondary school (age 12), since the curriculum includes related contents such as "History of life." On the other hand, the optional subject Biology and Geology (age 15) specifically includes the content "The first living beings."

\subsection{Draw an alien}

Most student drawings (46\%; Table 6) represented a generic human-type alien. In general, it had a head with antennae, two legs (although some had three), two arms, and hands with between three and five fingers. Other than these, two particular human-type movie aliens could be recognized among student drawings. These are "Paul," an alien type characterized by a

Table 5. Categories Defining Correct, Partially Correct, and Incorrect Answers to Item 3 of the Questionnaire

\begin{tabular}{lll}
\hline C & Bacteria type, prokaryotic cell & $23.6 \%$ \\
PC & Only specify they were unicellular & $46 \%$ \\
or microscopic & \\
I & Other, no answer, don't know & $30.3 \%$ \\
\hline
\end{tabular}

The right column indicates the percentage of student answers within each category.
Table 6. Classification of Answers to Item 4 in the Questionnaire

\begin{tabular}{|c|c|}
\hline Humanoid: & $\begin{array}{l}\text { Generic } \\
\text { "E.T." movie type } \\
\text { "Paul" movie type }\end{array}$ \\
\hline
\end{tabular}

The Simpsons octopus aliens (Kang and Kodos) type 7.9\% Amoeba type $\quad 5.6 \%$ Reasoned answer $\quad 9 \%$ Unclassifiable $\quad 6.7 \%$ Don't know, no answer $\quad 3.4 \%$

particularly big head and eyes that can be seen in $9 \%$ of the drawings. Some students pointed out that the alien depicted corresponds to the character from the movie titled Paul, a popular teen fi hence, these drawing were grouped under the "Paul" label. Another 9\% of the students drew an alien that looked like the E.T. character from the popular movie titled E.T. the Extra-Terrestrial. This character was also drawn by $9 \%$ of the students. However, it should be noted that a toy E.T. doll, unbeknownst to those administering the questionnaire, was present in the classroom while groups 1 and 2 answered the questionnaire, which probably increased the frequency for this category.

A total of $9 \%$ of the pupils chose not to draw an alien but indicated instead something akin to "How can I draw an alien if I have never seen a real one? At the most, I could draw a movie stereotype.' Answers such as these were assigned to the "reasoned answer" category.

Other recognizable aliens were those of the amoeba type, which can be seen in 5.6\% of the student drawings; and those of the octopus type, as popularized by The Simpsons television series, can be seen in $7.9 \%$ of the student drawings. Finally, $6.7 \%$ of the drawings were unclassifiable, in that they resembled animal forms. Two students indicated only that aliens would look "similar to those depicted in movies," and three seemed to have difficulty with the question-one indicating he or she did not know and two offering no answer.

\subsection{Bodies of the Solar System with biological interest}

A majority of students were able to name Mars as a body where there could have been life (PC; Table 7). A total of $55.1 \%$ explained that this is due to the presence of liquid water, although in some cases students were not clear as to whether this was in reference to the present or the past.

Table 7. Categories Defining Correct, Partially Correct, and Incorrect Answers to Item 5 of the Questionnaire

C Name at least two bodies where life could $1.1 \%$ exist or could have existed, and explain correctly why (either presence of water or suitable distance to the Sun)

PC Name one body with a correct explanation (here, all pointed to the existence of ice on Mars)

I No answer, don't know, or does not explain $41.6 \%$ why

The right column indicates the percentage of student answers within each category. 
Three students named the Moon, two of them indicating that "water has been found there" and another asserting that "life could be possible there by bringing air from Earth." Although the Moon is an inhospitable body for life, the water reasoning was considered correct.

Only one student correctly explained why two or more Solar System bodies have or have had favorable conditions for life (C). The student's argument was based on the habitable zone as follows: " ‘. maybe Mercury or other planets close to Sun, when the Sun was young, could have had water and maybe harbored life. In the future, when the Sun grows, it is possible that life could occur on Mars because it has solid water."

A total of $41.6 \%$ students were not able to mention a single Solar System body or give a correct explanation (I). This group consists mostly of students who indicated "Mars" but made no explanation as to why Mars or indicated an incorrect explanation as, for example, "Mars, because it is the nearest planet to Earth, and it has similar weather." Other incorrect answers included, for example, "As for Mars or Jupiter, given that they are almost at the same distance from the Sun as Earth, life might be a possibility."

In no case was Europa, Enceladus, Ganymede, or another potentially interesting body of the Solar System mentioned.

The official curriculum includes related topics, such as "Physical properties of Earth and other Solar System bodies," in the first year of secondary school (Natural Sciences, age 12).

\subsection{Whether life exists beyond the Solar System}

Most students were convinced of the existence of extraterrestrial life (Table 8). A total of $68.6 \%$ thought that life exists beyond Earth based on reasoned arguments (C). Among their arguments were the following: the infinite Universe (41.5\%); the adaptability of life to a wide variety of ambient conditions (6.7\%); the notion that if life emerged on Earth, it can emerge elsewhere (12.3\%), which can be interpreted as a version of the adaptability argument; and the notion that "we can't be the only living beings in the universe" (9\%), which can be interpreted as a reference to the infinite Universe as opposed to a hypothetical solitude in the Universe.

An additional $7.9 \%$ of the students indicated that life exists beyond Earth but provided no explanation (I), and another $2.2 \%$ also indicated that they believe in the existence of life but presented incorrect arguments (I), for example, "Yes, because universe is expanding" or "Everything is possible."

A total of $10.1 \%$ of the students did not believe that life exists anywhere other than on Earth and provided reasonable arguments (PC). Two types of arguments were put forth as follows: (i) despite the fact that we have searched for life

Table 8. Categories Defining Correct, Partially Correct, and Incorrect Answers to Item 6 of the Questionnaire

C Life probably exists elsewhere, with a correct $\quad 68.6 \%$ argument

PC Life probably does not exist elsewhere, with a correct argument

I No explanation, incorrect explanation, doubt, $21.3 \%$ no answer

The right column indicates the percentage of student answers within each category.
Table 9. Classification of Answers to Item 7 in the Questionnaire

\begin{tabular}{lr}
\hline Yes & $86.5 \%$ \\
No & $7.9 \%$ \\
Doubt & $3.4 \%$ \\
No answer & $2.2 \%$ \\
\hline
\end{tabular}

beyond Earth, it has not been found (6.7\%), or (ii) the rare Earth argument: it is problematic that another place exists with the same conditions that permitted the emergence of life on Earth $(3.4 \%)$.

Students who believed that there are no life-forms beyond the confines of Earth but offered no explanation or an incorrect explanation (I) represent $2.2 \%$ of the student answers. Students who doubted extraterrestrial life exists $(5.6 \%)$ or gave no answer $(2.2 \%)$ were also included in the incorrect category (I).

\subsection{The importance of discerning whether intelligent civilizations exist}

Most students (86.5\%; Table 9) thought that it was important to learn whether there are other intelligent civilizations in the Universe and provided a wide variety of arguments as to why, which included the following: for scientific or technical advancement $(53.9 \%)$; for an intrinsic interest toward other species $(11.2 \%)$; in the event humans must migrate away from Earth $(8.9 \%)$; to learn whether or not we are alone $(4.5 \%)$; to be prepared in the event we are invaded (2.2\%); if, at some point, we need more resources $(2.2 \%)$. On the contrary, $7.9 \%$ of the students did not consider knowledge of other intelligent civilizations to be important, some indicating that "we would destroy them" (3.4\%), others that "we have enough problems on Earth" (3.4\%). A total of $3.4 \%$ had difficulty with this question, answering they "didn't know," and $2.2 \%$ of the students did not respond.

\subsection{UFOs}

Student opinions on ufology were quite diverse (Table 10). A total of $31.5 \%$ of the pupils did not believe that aliens are visiting us and gave a correct justification (C), among them were the following: (i) "we would have already seen them" (20.2\%); (ii) "it has been investigated and discarded" (5.6\%); (iii) "it is an invention or a manipulation due to different interests" (3.3\%); (iv) "beings in our neighborhood of the galaxy are not evolved" (2.2\%); (v) "if they exist, they wouldn't be interested in us" (one person).

A large fraction of students answered that they did not know (21.3\%), which indicates that they have little clarity on

Table 10. Categories Defining Correct, Partially Correct, and Incorrect Answers to Item 8 of the Questionnaire

\begin{tabular}{llr}
\hline C & No, with a correct reasoning & $31.5 \%$ \\
PC & No, without argument or too vague & $22.4 \%$ \\
& Doubt & $21.3 \%$ \\
& No answer & $4.4 \%$ \\
I & Yes & $20.2 \%$ \\
\hline
\end{tabular}

The right column indicates the percentage of student answers within each category. 
Table 11. Categories Defining Correct, Partially Correct, and Incorrect Answers to Item 9 of the Questionnaire

C Name at least a project $3.4 \%$

PC Describe a project but do not remember $\quad 3.4 \%$ the name

I Don't know, no answer, incorrect

The right column indicates the percentage of student answers within each category.

this issue; others answered that they did not believe in ufology, but could not explain why (14.6\%); while others put forth an argument based on their personal experience as opposed to scientific or social arguments (6.7\%), such as "I have never seen one." A total of $4.4 \%$ of the students did not answer, which we interpreted as a sign of trepidation over this direct yes or no question. All these were classified as PC.

A non-negligible fraction of students (20.2\%) indicated belief in ufology (I). Within this group, three references to ancient Egypt and a reference to a Spanish paranormal television show were identified.

\subsection{Communication with other civilizations projects}

The attempts to establish communication with other possible civilizations, either by transmission or reception, represent the most unknown facts with regard to students from the questionnaire.

Only three students (3.4\%; Table 11) were able to mention the messages sent with the Voyager spacecrafts (C). Three other students $(3.4 \%)$ described the content of the message, though they did not remember the project name (PC).

The rest $(93.2 \%)$ were not able to name any project, or they put forth incorrect answers. Six students $(6.7 \%)$ offered "NASA" as an answer, as if the agency was by itself a project with the objective of establishing communication with other civilizations.

\subsection{Why are we in the Universe}

This question confounded students (Table 12). In fact, $21.6 \%$ of the students admitted that they did not know, $5.4 \%$ gave no answer, and $24.3 \%$ gave unclassifiable answers.

The most common answer was that we are in the Universe because of a series of random facts (41.9\%), followed by the argument that we are here "because there is no other place" $(20.3 \%)$. Marginally, three students made reference to the Big Bang (e.g., "We are here because of the Big Bang event'), while one student named destiny as the reason for our existence, and another named a god.

Table 12. Classification of Answers to Item 10 in the Questionnaire

\begin{tabular}{lr}
\hline Because of a series of random facts & $41.9 \%$ \\
Don't know & $21.6 \%$ \\
Unclassifiable & $24.3 \%$ \\
"Because there is no other place'" & $20.3 \%$ \\
No answer & $5.4 \%$ \\
Because of the Big Bang & $3.4 \%$ \\
Destiny or god & $2.2 \%$ \\
\hline
\end{tabular}

\section{Discussion}

In an earlier study (Oreiro and Solbes, 2015), textbooks were analyzed to review their astrobiology content. Most Spanish teachers use textbooks as everyday tools for teaching and try to cover their content throughout the academic year (Solbes et al., 2012). Therefore, the textbooks' quality and the way in which they incorporate the offi ial curriculum are directly linked to what students are taught. It was concluded that astrobiology is not well integrated into the Spanish curriculum. Some related topics are disconnectedly presented along different levels, but still many relevant issues are not addressed, which mostly have to do with exploration of the Solar System, the search for life beyond Earth and the Solar System, STS, and socio-scientific issues (Solbes and Vilches, 1997; Zeidler et al., 2005; Hansson et al., 2011).

Based on this, a particular student's performance would be expected to reflect that student's degree of exposure to aspects of astrobiology for each question. In some cases, as explained below, the results do not match such expectations.

One example of this is item 1, which concerns those factors that make possible the existence of life on Earth. This is, in fact, part of specific curriculum content in the first level of secondary school. However, by the time a student has reached the fourth level, as indicated in the present study, only $34.8 \%$ of the students are able to name at least three key factors.

Students in this study recognized water as a key factor for life, as it was named by $88.8 \%$ of them. There is a specific curriculum content- "The importance of water" - which is also presented in the first level of secondary school, that was soundly reflected in our results. This percentage is consistent with those (73-90\%) obtained by Hansson and Redfors (2013) to a more direct question (Is water necessary for life?) and by Offerdahl et al. (2003), depending on the student's age. Students were unable, however, to explain why water is fundamental for life in either of these studies.

In the present study, more than $50 \%$ of the students indicated that oxygen is a fundamental factor for life. This is a rather surprising result, as the primitive atmosphere, which was lacking in oxygen, is described in most textbooks for this academic level (Oreiro and Solbes, 2015). On other hand, textbooks do not include extremophiles, which would help students understand that some organisms do not depend on oxygen. Our result is significantly higher than the 16$25 \%$ (depending on age) published by Offerdahl et al. (2003), who proposed that students tend to think of complex organisms when asked about life.

Offerdahl et al. (2003) indicated that $22-41 \%$ of the students purported that life cannot exist in places that never receive sunlight. Hansson and Redfors (2013) reported a much higher percentage, about $90 \%$, to an equivalent question. In these studies, students were asked a direct yes or no question regarding the necessity of sunlight for life to exist. On the contrary, in our work students faced an open question that did not specifi ally ask about sunlight (see Table 1). With this proposal, a total of $31.4 \%$ of the answers included either "The Sun" or "Sunlight" among other necessary factors for life.

Half the students who referred to sunlight $(16.8 \%$ of the total student sample) also named oxygen as necessary for life. A possible connection between sunlight and oxygen may indicate a student's model in which sunlight is necessary for plants to produce oxygen and oxygen is necessary 
for life. This connection was noted by Offerdahl et al. (2003), since about $40 \%$ of their sample used this explanation to support the necessity of sunlight for life. A total of $22 \%$ of the students in the Hansson and Redfors (2013) study also justified sunlight as necessary for life because it is required for photosynthesis. Since our questionnaire did not specifically ask about these factors, the $16.8 \%$ of students who indicated a possible sunlight-oxygen interrelated model should be considered as a lower limit.

A optimal distance from a star as an important factor for life was named only in $20.2 \%$ of the cases, which is in agreement with the lack of the habitable zone description in textbooks.

With regard to item 2, very few students $(23.6 \%)$ answered correctly as to when life emerged on Earth. This topic appears in the official curriculum in the first and the fourth levels of secondary school. Natural Sciences (age 12) includes the item "History of life," while Biology and Geology (age 15) includes the items "Earth history" and "The origin of life." Furthermore, this item is well explained in textbooks (Oreiro and Solbes, 2015); hence, a better performance was expected. As possible explanations for this we consider the following: (i) in many cases this issue is included at the end of textbooks and may not always be taught; (ii) the lack of timescale exercises that could help better integrate greater scale times (Cheek, 2012); (iii) textbooks normally do not include the evidence upon which the origin of life is estimated, and thus students could view the date information simply as something established, which could be lacking in meaning for them.

In the work by Marques and Thompson (1997), about half the students that participated believed that the origin of Earth and life occurred simultaneously. In the present work, only two students stated it this way (e.g., I think [life started] approximately at the same time the Earth was formed), and no students expressed that life started before the formation of Earth.

Students also had problems explaining how life began. Despite the fact that origins of life is a common subject in textbooks, only $15.7 \%$ of the students referred to one plausible theory. The difficulties students show here should be further investigated.

The first living beings on Earth are also poorly known among students: less than a quarter of students correctly answered this question $(23.6 \%)$. The most common answer to this question was that the first forms of life were either unicellular or microscopic (PC). A high percentage (30.3\%) did not answer or answered incorrectly. Among incorrect answers, we point out a fraction $(12 \%)$ that cannot be neglected, which indicated that the first living creatures were either apes, dinosaurs, plants, or Adam and Eve. Here, a better result was expected, given that, first, the description of the first living beings on Earth is included in the official secondary education curriculum, and second, it is also included in textbooks used in the fourth level of secondary school (Oreiro and Solbes, 2015).

When students were asked to draw an alien, $64 \%$ of them refl ed a humanoid type, either a generic one or an "E.T." or "Paul" leading actor. This percentage is significantly higher than the $20 \%$ of college students who responded in a study by LoPresto and Hubble-Zdanowski (2012) that aliens would not necessarily take human form, although the students' age may be playing an important role. Only $9 \%$ of the students that participated in the present study gave reasoned answers.

Only one student in our study gave a correct answer with regard to Solar System bodies with favorable conditions for life. A total of $57.3 \%$ of the students knew that Mars is one of the most interesting bodies from a biological point of view, and they were able to explain why. However, a high percentage $(41.6 \%)$ either did not respond or responded that Mars is interesting but could not explain why. This scarce knowledge of the Solar System, particularly from a biological perspective, agrees with the lack of this kind of content in textbooks. This is striking considering that properties of the Solar System constitute a reiterative astronomy curriculum content from primary to secondary school. This might indicate that properties such as mass, size, temperature, or distance with regard to bodies are correctly explained in textbooks but that recent findings as a result of Solar System exploration, particularly regarding water abundance and potential conditions for life, have not yet been incorporated into the formal education system in Spain. The impact that informal education might have on this particular issue is worth investigating.

Most students believe that life exists beyond Earth, and they are able to offer correct arguments $(68.5 \%)$ that are related to the infinite Universe argument, though this is contrary to the $10.1 \%$ of students who did not believe in life outside our planet and gave correct arguments. The remainder, a total of $21.3 \%$, did not answer the question or answered "I don't know" to the question of whether they think life exists beyond Earth, or did not explain why they were either in favor of or against the idea of life beyond the confines of Earth; in short, these students were not able to define their position on such a fundamental question.

Despite these concerns, a wide majority (86.5\%) thought that it is important to discern whether there is intelligent life in other parts of the Universe. The main argument here was that such a discovery would mean a major scientific or technological advance for us, as explained by $53.9 \%$ of the students. This may imply that science and technology are viewed favorably by most students, and they consider such advancements as important. On the contrary, $7.9 \%$ of the students thought that it was not important to find other civilizations. As suggested by Hansson et al. (2011), debates on socio-scientific issues, in particular whether we should look for extraterrestrial life, enhance the use of scientific arguments in decision making and, hence, should be regarded as important tools for general science teaching.

With respect to UFOs, $31.5 \%$ of the students did not believe in their existence and could explain why, while $20.2 \%$ confessed that they believed UFOs are visiting our planet. These numbers agree with the average result obtained by an international poll (Ipsos, 2010), although the percentage is lower than in other countries (Ipsos, 2015). But the most remarkable fact is the high number of students (48.3\%) who did not define their position, as follows: (i) they stated that they did not have this issue clear, (ii) they did not respond, or (iii) they were not able to elaborate any argument against ufology. Ufology is not discussed in textbooks, and for this reason a potential opportunity to exercise critical thinking in the classroom has been lost.

Despite their interest in unveiling the possible existence of life beyond Earth, students showed a very real lack of 
awareness of historical or present attempts to establish communication with, or search for, extraterrestrial intelligence, given that $93.2 \%$ were not aware of any project with this aim. This, however, is an expected result, since this content is not found in commonly used textbooks. This appears to be a missed opportunity for an astrobiological contribution to an integral education, as this content not only informs from a historical perspective but also with regard to science-technology-society relations. And it would seem that the null results of these kinds of projects could represent an argument against ufology.

The last item of the questionnaire (Why do you think we are in the universe?) generated very divergent answers, probably due to the open proposal. A total of $41.9 \%$ of the answers were of the "Due to a series of random facts" kind of answer, while $25 \%$ of the students gave a variety of answers that were difficult to classify. Approximately another $25 \%$ did not know an answer or chose not to answer. Some students said that they "had never thought about it" or that " nobody had asked something like this before." This indicates the difficulties students have in argumentation, the lack of training in philosophical implications of science, and the need to include scientific debates in the classroom. On the other hand, no signs of creationism were detected in the students' answers, and only one student made reference to a divine explanation for our existence.

\section{Conclusions}

A questionnaire to explore students' views on astrobiology was distributed to a sample of 89 students in their last year of Spanish compulsory school (age 15). This effort was undertaken to add to the small number of previous investigations on this matter (Offerdahl et al., 2003; Hansson and Redfors, 2013) with the expectation that it might help in the design or improvement of a contemporary astrobiology curriculum.

Our results reveal major diffi lties students have concerning concepts in astrobiology. In particular, students had difficulty describing environmental conditions that are necessary for life. Few students could name at least three factors that would have made life possible on Earth. Many thought that oxygen and sunlight were necessary for life to exist, which may indicate that students tend to consider complex organisms rather than the wide range of living forms that exist on Earth, particularly microorganisms, when discussing life.

Few students could indicate when life began on Earth; many, rightfully so, had difficulty explaining how it started; and a majority of students demonstrated a lack of knowledge as to what the first living forms that appeared on Earth were. It should be noted here that these topics on early life are covered in textbooks used by the students who participated in this study.

We also found that less than $60 \%$ of the students could explain why Mars is an interesting planet from a biological perspective, which is surprising considering the present popularity of Mars exploration. Moreover, students were unable to think of other Solar System bodies that would have favorable conditions for life, indicating that these kinds of advances in science are not being incorporated into formal primary and secondary education, which could produce a literacy gap between science and citizens.

Most students $(68.5 \%)$ believed that life exists beyond Earth and were able to defend their position with correct arguments. Also, a high majority $(86.5 \%)$ considered it important to discern whether there is intelligent life in other parts of the Universe. This may imply that students support research with this aim, despite the fact that these questions are not included in textbooks and hence are not worked into the Spanish formal education.

Our results also indicate the necessity that debates be included in scientific formal education. Taking advantage of the interest astrobiology seems to garner worldwide, the discipline could serve as a framework to enhance student skills in decision making or reasoning with regard to philosophical or socio-scientific discussions. In the present study, students had difficulty defending their positions on issues such as the potential for life beyond Earth, whether it is important to discern it, whether UFOs are real, or why we exist in the Universe. This seems to indicate that the philosophical implications of science, together with STS and socioscientific issues, are not a part of primary and secondary science lessons.

Historical aspects related to astrobiology are also largely forgotten in textbooks and secondary level science classes in general. The sample of students were unaware of past or present efforts to search for signs of other intelligent civilizations.

These conclusions, along with those of existing works (e.g., Carrapiço et al., 2002; Brake et al., 2006; Slater, 2006; Arino de la Rubia et al., 2009; Oliveira and Barufaldi, 2009; Quinlan, 2015), will be considered for the implementation of an astrobiology curriculum for secondary school students within the Spanish educational system.

\section{Acknowledgments}

The authors thank the Sky Classroom people, from the Astronomical Observatory in the University of Valencia, particularly M. Gómez, X. Moya, A. Ortiz Gil, and F. Ballesteros for their help and kindness.

\section{Author Disclosure Statement}

No competing financial interests exist.

\section{References}

Arino de la Rubia, L., Butler, J., Gary, T., Stockman, S., Mumma, M., Pfiffner, S., Davis, K., and Edmonds, J. (2009) Development, evaluation, and dissemination of an astrobiology curriculum for secondary students: establishing a successful model for increasing the use of scientific data by underrepresented students. In Bioastronomy 2007: Molecules, Microbes, and Extraterrestrial Life, ASP Conference Series Vol. 420, Astronomical Society of the Pacific, San Francisco, pp 471-476.

Brake, M., Griffiths, M., Hook, N., and Harris, S. (2006) Alien worlds: astrobiology and public outreach. International Journal of Astrobiology 5:319-324.

Briot, D. (2013) Elements for the history of a long quest: search for life in the Universe. International Journal of Astrobiology 12:254-258.

Burchell, M. and Dartnell, L. (2009) Astrobiology in the UK. Astronomy and Geophysics 50:27-30.

Carrapiço, F., Lourenço, A., Fernandes, L., and Rodrigues, T. (2002) A journey to the origins. The astrobiology paradigm in education. Proc SPIE 4495:295-300. 
Cheek, K.A. (2012) Students' understanding of large numbers as a key factor in their understanding of geologic time. International Journal of Science and Mathematics Education 10:1047-1069.

Des Marais, D.J., Nuth, J.A., III, Allamandola, L.J., Boss, A.P., Farmer, J.D., Hoehler, T.M., Jakosky, B.M., Meadows, V.S., Pohorille, A., Runnegar, B., and Spormann, A.M. (2008) The NASA Astrobiology Roadmap. Astrobiology 8:715-730.

Dinov, I.D. (2008) Integrated, multidisciplinary and technologyenhanced science education: the next frontier. J Online Learn Teach 4:84-93.

Fergusson, J., Oliver, C., and Walter, M.R. (2012) Astrobiology outreach and the nature of science: the role of creativity. Astrobiology 12:1143-1153.

Griffiths, M. (2004) Broad horizons-SETI, SF and education. International Journal of Astrobiology 3:175-181.

Hansson, L. and Redfors, A. (2013) Lower secondary students' views in astrobiology. Res Sci Educ 43:1957-1978.

Hansson, L., Redfors, A., and Rosberg, M. (2011) Students' socio-scientific reasoning in an astrobiological context-the design and implementation of a digital learning environment. J Sci Educ Technol 20:388-402.

Ipsos. (2010, April 8) One in Five (20\%) Global Citizens Believe That Alien Beings Have Come Down to Earth and Walk Amongst Us in Our Communities Disguised as Humans, Ipsos North America, New York. Retrieved from http://www.ipsosna.com/news-polls/pressrelease. aspx ?id=4742

Ipsos. (2015, June 29) Americans Pass Judgment on the Plausibility UFO's, Extraterrestrial Visits and Life Itself, Ipsos North America, New York. Retrieved from http://www.ipsosna.com/news-polls/pressrelease. $\operatorname{aspx}$ ?id=6902

LoPresto, M.C. and Hubble-Zdanowski, J. (2012) A life in the Universe survey. Astronomy Education Review 11, ID: 010110.

Marques, L. and Thompson, D. (1997) Portuguese students' understanding at ages $10-11$ and 14-15 of the origin and nature of the Earth and the development of life. Research in Science and Technological Education 15:29-51.

Mix, L.J., Cameron, V., Claire, M., Dick, G.J., Domagal-Goldman, S.D., Javaux, E.J., Johnson, O.J., Laws, C., Race, M.S., Rask, J., Rummel, J.D., Schelble, R.T., and Vance S. (2006) The Astrobiology Primer: an outline of general knowledge. Astrobiology 6:735-813.

NASA. (2006) Astrobiology in Your Classroom: Life on Earth . and Elsewhere? NASA Ames Research Center, Moffett Field, CA.

Offerdahl, E., Prather, E.E., and Slater, T.F. (2003) Students' pre-instructional beliefs and reasoning strategies about astrobiology concepts. Astronomy Education Review 1:5-27.

Oliveira, C. (2008) Astrobiology for the $21^{\text {st }}$ century. Communicating Astronomy with the Public 2:24-25.

Oliveira, C. and Barufaldi, J.P. (2009) Aliens are us. An innovative course in astrobiology. International Journal of Astrobiology 8:51-61.

Oreiro, R. and Solbes, J. (2015) Evaluación de la enseñanza de la Astrobiología en secundaria: análisis de libros de texto y opiniones del profesorado en formación. Didáctica de las Ciencias Experimentales y Sociales 29:247-274.

Ortiz Gil, A., Gómez Collado, M., and Gallego Calvente, A.T (2005) L'aula del cel. In Communicating Astronomy with the Public: 2005, edited by I. Robson, L. Lindberg Christensen, and M. Kommesser, Garching bei München, Germany, pp 358-359. Quinlan, C.L. (2015) Bringing astrobiology down to Earth. Am Biol Teach 77:567-574.

Rodrigues, T. and Carrapiço, F. (2005) Teaching astrobiology: a scientific and cultural imperative. Proc SPIE 5906, doi:10 $.1117 / 12.617594$

Rodrigues, T. and Carrapiço, F. (2006) How can we teach astrobiology and survive? Proc SPIE 6309, doi:10.1117/12 .681950 .

Slater, T.F. (2006) Capturing student interest in astrobiology through dilemmas and paradoxes. $J$ Coll Sci Teach 35:42-45.

Solbes, J. and Vilches, A. (1997) STS interactions and the teaching of physics and chemistry. Sci Educ 81:377-386.

Solbes, J., Furió, C., Domínguez, M.C., Fernández, J., Tarín, F., and Guisasola, J. (2012) What factors have an influence on a quality teaching practice in sciences? Procedia Soc Behav Sci 46:4513-4517.

Staley, J.T. (2003) Astrobiology, the transcendent science: the promise of astrobiology as an integrative approach for science and engineering education and research. Curr Opin Biotechnol 14:347-354.

Tang, B.L. (2005) Astrobiological themes for integrative undergraduate general science education. Astronomy Education Review 4:110-114

Zeidler, D.L., Sadler, T.D., Simmons, M.L., and Howes, E.V. (2005) Beyond STS: a research-based framework for socioscientific issues education. Sci Educ 89:357-377.

Address correspondence to: Jordi Solbes Science Education Department Valencia University Tarongers Av., 4 46022 Valencia Spain

E-mail: Jordi.solbes@uv.es

Submitted 10 December 2015 Accepted 13 December 2016 Abbreviations Used

C $1 / 4$ correct

$\mathrm{I} 1 / 4$ incorrect

$\mathrm{PC} 1 / 4$ partially correct

STS $1 / 4$ science and technology studies 